\title{
Soft Switching Behavior of IGCT for Resonant Conversion
}

D. Stamenkovic, U. Vemulapati, M. Rahimo, et al.

This material is posted here with permission of the IEEE. Such permission of the IEEE does not in any way imply IEEE endorsement of any of EPFL's products or services. Internal or personal use of this material is permitted. However, permission to reprint / republish this material for advertising or promotional purposes or for creating new collective works for resale or redistribution must be obtained from the IEEE by writing to pubs-permissions@ieee. org. By choosing to view this document, you agree to all provisions of the copyright laws protecting it. 


\title{
Soft Switching Behavior of IGCT for Resonant Conversion
}

\author{
Dragan Stamenkovic ${ }^{1}$, Umamaheswara Reddy Vemulapati ${ }^{2}$, Thomas Stiasny ${ }^{2}$, Munaf Rahimo ${ }^{3}$ and Drazen Dujic ${ }^{1}$ \\ ${ }^{1}$ Power Electronics Laboratory, École Polytechnique Fédérale de Lausanne, Station 11, Lausanne, Switzerland \\ Email: dragan.stamenkovic@epfl.ch,drazen.dujic@epfl.ch \\ ${ }^{2}$ ABB Semiconductors, Fabrikstrasse 3, Lenzburg, Switzerland \\ Email: umamaheswara.vemulapati@ch.abb.com, thomas.stiasny@ch.abb.com \\ ${ }^{3}$ MTAL GmbH, Unterer Schafmattweg 39A, Gaensbrunnen SO, Switzerland \\ Email: munaf.rahimo@mtal.ch
}

\begin{abstract}
Application of the Integrated Gate Commutated Thyristor as a switching element in the medium voltage series resonant converter for DC-DC conversion offers the opportunity for increased conversion efficiency and higher switching frequency of the converter. Low current turn-off, already discussed in the previous works, shows promising results for increasing operating frequency of the converter, thus enabling the use of physically smaller components in the design, decreasing the total volume and weight of the solution. This paper presents the results of the switch characterization under single resonant current pulse operation, providing further insight into the turn-off process, relevant timing intervals and resulting losses, supported by TCAD simulations and experimental results.
\end{abstract}

\section{INTRODUCTION}

Increased interest in Medium Voltage Direct Current (MVDC) power distribution has been observed lately, driven by the marine sector [1], [2] and followed by the work done considering high power distribution for solar [3] and wind farms [4], [5]. Main motivation behind reviving the DC distribution lies in increased efficiency of power distribution networks and reduced number of voltage conversions in the system and, as a consequence, lower number of equipment elements needed for the system operation. Especially in the renewable energy generation sector, where many stages of electrical-to-electrical power conversion are needed in order to feed the energy into the AC grid.

Key missing element for the wide application of the MVDC distribution is the DC transformer able to adjust the voltage levels between various parts of the system while offering galvanic isolation among them. Considerable work has been done regarding the Solid State Transformer (SST), power converter intended to overtake the role of the Low Frequency Transformer (LFT) found within modern Alternating Current (AC) power systems. Nevertheless, commercial products of this kind are currently not available and significant research and development work is needed in this area.

Various converter topologies are proposed as circuit of choice for the SST ([6]-[8]), with multi-stage conversion including AC-DC, DC-DC and DC-AC, not necessarily in this order. Intermediate AC conversion stage is usually implemented using the Medium Frequency Transformer (MFT) which offers flexible voltage transformation and galvanic isolation between input and output stages of the converter and operation at medium frequency ensures the smaller volume and lower weight when compared to the conventional $50 \mathrm{~Hz} / 60 \mathrm{~Hz}$ transformers.

In the area of MVDC DC-DC conversion, two topologies, Dual-Active Bridge (DAB) [8] and Series Resonant Converter (SRC) [4], [9] with their single and three-phase variants, emerge as the most popular throughout industry and academia. Owing it to its resonant mode of operation, generally lower switching losses and less stringent control SRC is emerging as the topology that offers increased efficiency, reliability and flexibility [5].

Insulated Gate Bipolar Transistor (IGBT) is usually primary technology used because of its popularity and availability. Alternative to the IGBT in the area of medium voltage high current switching can be found in the Integrated GateCommutated Thyristor (IGCT) type of device, a mature technology found in many applications ([10], [11]) offering various advantages over IGBT [12], [13]. Being a thyristor-based device, conduction losses are significantly lower compared to the IGBT of the similar rating; due to the mandatory $\mathrm{di} / \mathrm{dt}$ limiting inductor in the clamping circuit, turn-on losses are practically zero (zero-voltage switching) while the turn-off losses are comparable to that of IGBT. Additional beneficial features offered by the IGCT, such as high short circuit capability, high reliability and large Safe Operating Area (SOA), result in higher current carrying capability compared to the IGBT of the same voltage rating [14], [15].

Implementing IGCT in the SRC presents the opportunity to further improve the performance of the final power electronics converter. Low current turn-off, discussed in [16] suggests that by properly tailoring the SRC turn-off current, one can obtain a design with low turn-off losses which presents the opportunity to increase switching frequency of the converter, thus decreasing the size of the surrounding passive components. Conduction losses of the IGCT are already lowest among the switches of the similar rating, owing it its thyristor nature. Resonant operation and soft turn-on naturally limit the $\mathrm{di} / \mathrm{dt}$ in the anti-parallel diodes which requires revising a role and necessity of the clamping circuit.

This paper provides further insight into operation of the 


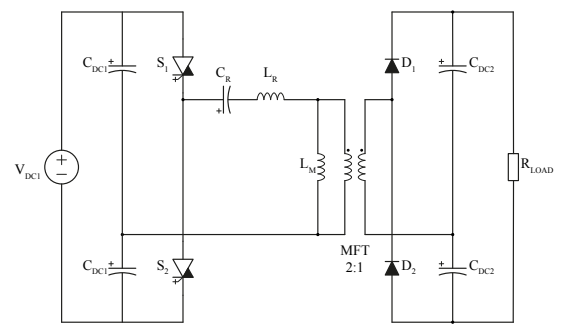

(a)

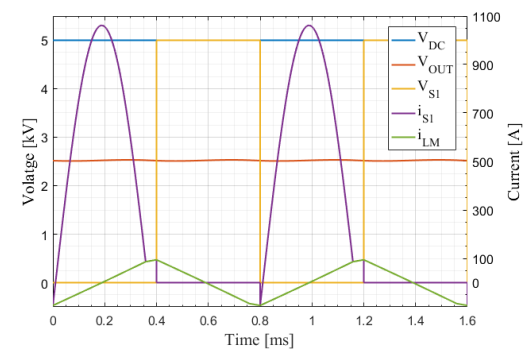

(b)

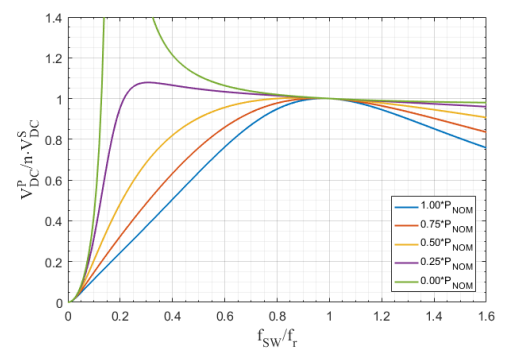

(c)

Figure 1: a) Half bridge SRC b) SRC characteristic waveforms c) SRC transfer characteristic.

IGCT under resonant switching conditions with higher frequencies of operation than presented in [17]. Measured turnoff delay times and turn-off losses of the device under test are presented including results obtained by means of Technology CAD (TCAD) and further verification on the characterization test setup.

\section{Series Resonant Converter}

Fig. 1a shows the half-bridge SRC topology with resonant circuit consisting of the resonant capacitor and the leakage inductance of the MFT. Design parameters for the given example (Fig. 1a) are presented in Table I and are based on the transformation ratio of 2 with the rated power of $1.5 \mathrm{MW}$. Typical waveforms for the primary side switches and the transfer characteristic are shown in Fig. 1b and Fig. 1c respectively. Looking into transfer characteristic, Fig. 1c, it can be noted that in the area of switching to resonant frequency ratio near 1 , converter output voltage remains relatively independent of the load and no control is required to maintain the output voltage relatively stable. With switching frequency selected to be lower than resonant, constant current turn-off of the IGCT is achieved which results in nearly constant turn-off energy losses of the switch throughout the working range of the converter. By tailoring the magnetizing inductance of the MFT, IGCT turn-off current can be finely tuned for minimal turn-off energy losses and safe turn-off behavior.

Referring to the shape of electrical current of the switch $S_{1}$ in Fig. 1b, it can be noted that it is negative at the beginning of the conduction period. While the current is negative, it is being supported by the anti-parallel diode of the $S_{1}$ and the IGCT of $S_{1}$ should be turned on during this period in order to be ready to take over the current when it becomes positive. This way, the voltage across the IGCT is kept low (forward voltage of the diode) providing the zero-voltage turn-on, leading to zero turn-on energy losses whatsoever.

\begin{tabular}{rl}
\multicolumn{2}{c}{ Table I: SRC Parameters } \\
\hline Parameter & Value \\
\hline$V_{D C 1}, V_{D C 2}$ & $5000 \mathrm{~V}, 2500 \mathrm{~V}$ \\
$C_{R}, L_{R}$ & $80 \mu F, 167 \mu H$ \\
$L_{M}$ & $5 \mathrm{mH}$ \\
$C_{D C 2}$ & $5.64 \mathrm{mF}$ \\
$R_{L O A D}$ & $4.17 \Omega$ \\
$f_{R}, f_{S W}$ & $1375 \mathrm{~Hz}, 1250 \mathrm{~Hz}$ \\
\hline
\end{tabular}

$S_{1}$ diode conduction time $t_{D O N}$ is defined as the time needed for the diode current to fall to zero i.e. time during which the $S_{1}$ current is negative. This parameter depends on the magnetizing inductance of the MFT, peak of the resonant tank current (directly proportional to the load of the converter), resonant and switching frequencies. The minimal value of $t_{D O N}$ under all working conditions of the converter is the maximum limit for the dead-time to be applied in the bridge.

Minimum limit for the dead-time is dictated by the time needed for the IGCT to fully turn off i.e. to fully take over the DC-link voltage. This time period is called turn-off delay time $t_{D O F F}$, defined by the manufacturer as the period of time between the moment of application of the turn-off signal to the IGCT's gate driver input until the moment when the current through the IGCT falls to $40 \%$ of the conduction current and it is provided in the datasheet of the IGCT for the worst case scenario of the switch operation. It is presented in [16] that $t_{D O F F}$, under hard-switching, strongly depends on the turnoff current and the temperature of the device and the results in [17] suggest that $t_{D O F F}$ is not affected by the resonant operation of the IGCT (as found in SRC).

As the $t_{D O F F}$ graphs are not present in the datasheet of the IGCT, especially not for turn-off at low current levels and resonant operating conditions, it is necessary to further characterize the device and understand its behavior within the SRC operating environment. In order to accomplish this goal, TCAD model of the IGCT was used to gain the initial results, followed by the experiments performed on a dedicated physical test setup.

\section{TCAD Simulation Results}

The tool based on the finite element method for solving the fundamental partial differential equations representing the silicon wafer in a semiconductor device (e.g. transport and Table II: Circuit parameters for resonant operation

\begin{tabular}{rl}
\hline Parameter & Value \\
\hline$V_{D C}$ & $1250 \mathrm{~V}$ \\
$L_{S}$ & $400 \mathrm{nH}$ \\
$D_{F W}$ & Ideal diode \\
IGCT & 5 SLD 0600J65010 \\
$I_{C M D}\left(0 \leq t \leq \frac{T_{r}}{2}\right)$ & $I_{M} \sin \left(2 \pi f_{r} t\right)+2 f_{S W} I_{O F F} t$ \\
$I_{C M D}\left(\frac{T_{r}}{2} \leq t \leq \frac{T_{S W}}{2}\right)$ & $2 f_{S W} I_{O F F} t$ \\
$f_{S W}, f_{r}$ & $4200 \mathrm{~Hz}, 4600 \mathrm{~Hz}$ \\
\hline
\end{tabular}




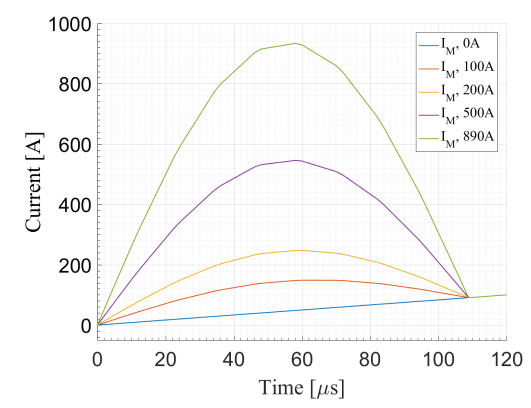

(a)

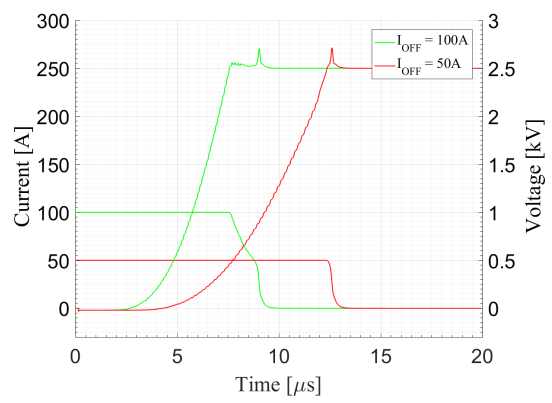

(b)

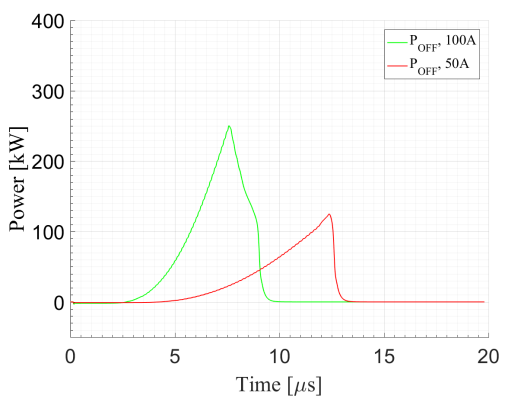

(c)

Figure 2: a) IGCT current with different maximum resonant current peaks and constant turn-off current of $100 \mathrm{~A}, f_{S W}=4200 H z$ b) IGCT current and voltage waveforms during turn off at 50A and 100A turn-off current; resonant current peak has no influence on the turn-off period and all current-voltage pairs look the same c) Transient turn-off power losses; turn-off energy lost is calculated by integrating power curves and in this case $E_{O F F}(50 A)=0.38 J, E_{O F F}(100 A)=0.67 J$

diffusion equations), Sentaurus TCAD simulation package, was used to obtain initial results of the IGCT switching behavior under relatively high switching frequency. The model was prepared and provided by the industrial partner with the geometry and parameters set to correspond to the actual device, commercial product, used in the test setup as a device under test. SPICE based description of the circuit coupled with the TCAD model of the device was employed in this kind of simulation and the initial turn-off waveforms are obtained. Experiments are performed under the same operating conditions.

IGCT is simulated with the circuit environment that corresponds to the operation of the typical SRC (Fig. 3) with the parameters set according to Table II, while the model of the semiconductor is simulated in the finite element manner. Conduction current through the IGCT is dictated by the current source $I_{C M D}$ consisting of the positive sinusoidal pulse, simulating resonant current of the tank, superimposed on a linear function of time presenting the magnetizing current of the MFT. Leakage inductance of the bus-bar is modeled as an inductor $L_{\sigma}$ and its value is set based on a value measured in the test setup.

Simulation data presented assume IGCT operation under switching frequency of $f_{S W}=4200 \mathrm{~Hz}$ and resonant frequency of $f_{R}=4600 \mathrm{~Hz}$. Maximum value of the resonant current is varied $(0 A, 100 A, 200 A, 500 A$ and $890 A)$, as well as the value of the turn-off current $(50 A, 100 A)$ to show the influence of these values on the turn-off process in terms of turn-off power losses and duration of the turn-off process. Fig. 2a shows the shape of current through IGCT before the turnoff signal is applied, with turn-off current value set to $100 \mathrm{~A}$. The same simulation is performed for the turn-off current of $50 \mathrm{~A}$ and the transient behavior of the current and voltage after the turn-off signal is applied, for both $50 \mathrm{~A}$ and $100 \mathrm{~A}$ turn-off, are shown in Fig. 2b.

Simulation shows that the shape of turn-off transient is independent of the peak resonant current through the IGCT or its shape i.e. there is no difference in the waveforms between the current ramp (resonant current part equal to zero) and the sum of the current ramp and resonant current.

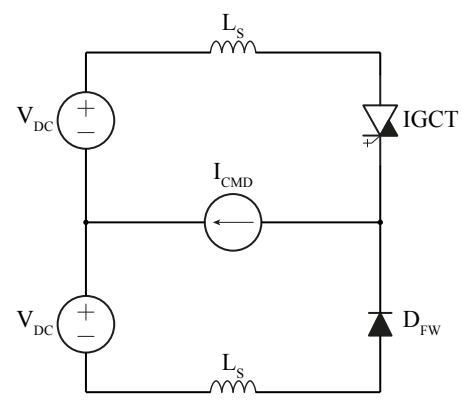

Figure 3: SPICE circuit implemented around the TCAD model of the IGCT used to define the shape of load current of the switch before the turn-off event according to the parameters shown in Table II

Further implication of this result is that the double pulse test parameters regarding the turn-off transient can be used for the resonant operation as well. Similar results are presented in [17] where a lower switching frequency is considered.

Fig. $2 b$ also depicts the major influence of turn-off current on the duration of the turn-off process. Turn-off delay times for the two switching currents are:

$$
t_{D O F F}(50 A)=12.5 \mu s, t_{D O F F}(100 A)=8.9 \mu s
$$

It should be noted that the same results are presented in [16] where double pulse test was considered which is another argument towards the conclusion that the shape of the conduction current does not influence the IGCT operation at turn-off. Comparing to results presented in [17], switching and resonant frequency don't significantly influence the shape and duration of the turn-off process.

Finally, Fig. 2c shows the transient turn-off power losses of the IGCT from which turn-off switching energies can be calculated as the area under the curve. Calculated values for the two turn-off currents are:

$$
E_{O F F}(50 A)=0.38 J, E_{O F F}(100 A)=0.67 J
$$

The turn-off energy parameters can further be used to evaluate the turn-off switching losses for the final design of the converter at the desired frequency of operation. Referring to the Section, TCAD model can be used to gain the initial values for the $t_{D O F F}$ and $E_{O F F}$ to be used in dead-time and power losses evaluation for the SRC design. 


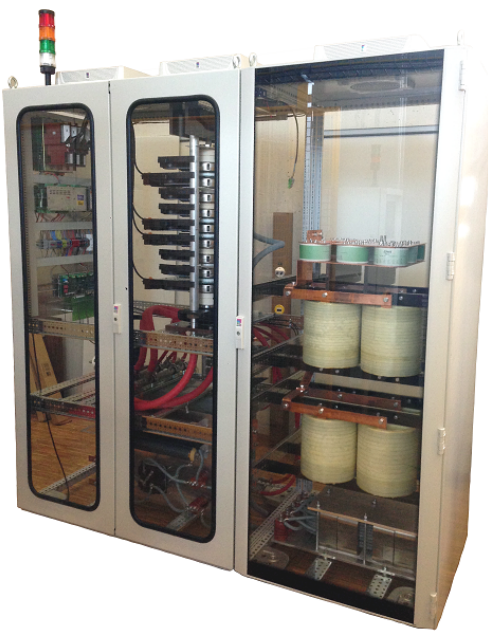

Figure 4: IGCT test setup

\section{Experimental Results}

Fig. 4 shows the implementation of the test setup assembled for the IGCT testing purposes in both hard switched (e.g. double pulse test) and resonant operation modes, while Fig. 6 presents the equivalent circuit of the test setup configured for the resonant operation. In order to set the switching frequency of interest, turn-on time of the device under test is manipulated while discretely varying the $\mathrm{L}_{\mathrm{R}}(7.5 \mu \mathrm{H}, 15 \mu \mathrm{H})$ and $\mathrm{C}_{\mathrm{R}}(80 \mu \mathrm{F}, 160 \mu \mathrm{F}, 640 \mu \mathrm{F})$ in order to change the resonant frequency of the tank. $\mathrm{L}_{\mathrm{R}}$ is a discrete component in the test setup and it emulates the leakage inductance of the MFT in the actual SRC while $\mathrm{L}_{\mathrm{M}}$ emulates the magnetizing inductance of the same MFT.

Test procedure normally involves selection of the resonant tank parameters for the desired resonant frequency and connecting the defined $L_{R}$ and $C_{R}$ components into the main circuit. Depending on the desired level of the turn-off current, $\mathrm{L}_{\mathrm{M}}$ inductor value is selected and connected into the circuit as well. After the manipulation of the components is done, precharging of the DC-link to the desired test value is executed, $2.5 \mathrm{kV}$ in this case. Next, capacitor $\mathrm{C}_{R}$ is charged to the voltage level corresponding to the peak of the resonant current that should be achieved, and can be calculated using the formula:

$$
I_{M}=U_{C 0} \sqrt{\frac{C_{R}}{L_{R}}}
$$

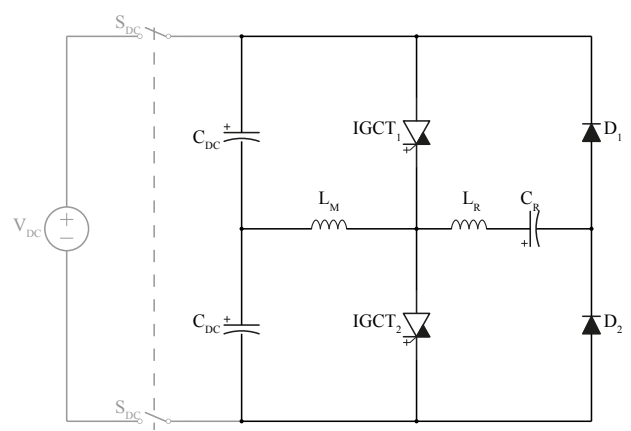

Figure 6: Resonant operation equivalent circuit of the test setup

When the $C_{R}$ reaches the desired voltage, IGCT $_{2}$ is turned on for the period of time corresponding to the half period of the test switching frequency. During this time, current through the inductor $\mathrm{L}_{\mathrm{M}}$ is being build up, and the resonance occurs in the circuit including $\mathrm{C}_{\mathrm{R}}, \mathrm{L}_{\mathrm{R}}, \mathrm{IGCT}_{2}$ and $\mathrm{D}_{2}$. Current through the device under test $\left(\mathrm{IGCT}_{2}\right)$ is the sum of resonant current and the $\mathrm{L}_{\mathrm{M}}$ inductor current. Short after the resonance is finished and only $\mathrm{L}_{\mathrm{M}}$ current flows through $\operatorname{IGCT}_{2}\left(f_{S W}<f_{R}\right)$, turn-off command is executed. $\mathrm{IGCT}_{2}$ current commutates to the anti-parallel diode of the $\mathrm{IGCT}_{1}, \mathrm{~L}_{\mathrm{M}}$ is connected to the opposite half of the DC-link voltage and its current starts falling towards zero, after which the test is done and can be repeated later under different conditions.

Current and voltage waveforms are recorded using high bandwidth transducers and high resolution oscilloscope for further analysis and parameter extraction. Temperature of the device under test is dictated by the cooling fluid (de-ionized water) which was kept at the temperature of $30^{\circ} \mathrm{C}$ during all of the tests. Influence of the leakage inductance of the commutation loop was not considered in the experiment and remained as designed among the different measurement sets.

Fig. 5 shows one current pulse of the IGCT for the different predefined turn-off currents, switching/resonant frequencies and peak resonant currents. In this set of experiments, only $\mathrm{IGCT}_{2}$ is pulsed and its current starts from zero. Resonant current amplitudes chosen for the test are 100A, 200A, 500A and $890 A$ so that the maximum turn-off current of the IGCT under test is never exceeded. Pulse lengths for the different resonant frequencies are set to $250 \mu \mathrm{s}, 170 \mu \mathrm{s}$ and $120 \mu \mathrm{s}$, cor-

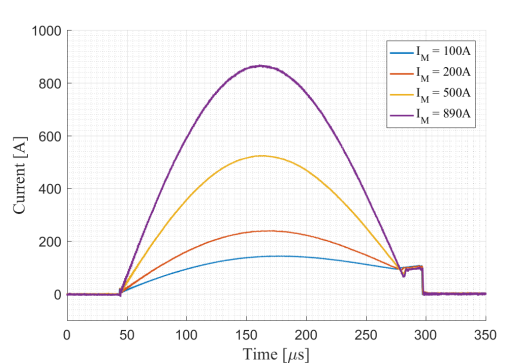

(a)

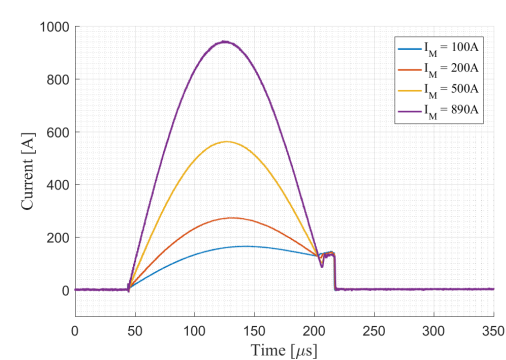

(b)

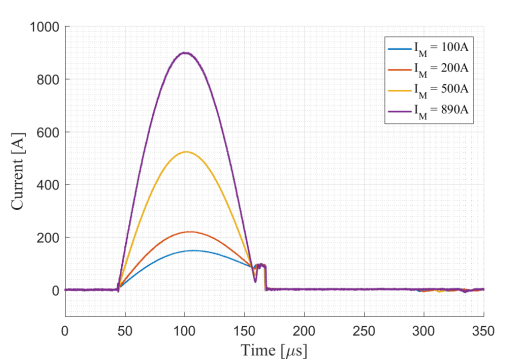

(c)

Figure 5: Measurement results for the three different resonant frequencies, variable peak resonant current and constant turn-off current a) $f_{R}=2300 H z$, $I_{O F F}=100 \mathrm{~A}$ b) $f_{R}=3250, I_{O F F}=140 \mathrm{~A}$ c) $f_{R}=4600 \mathrm{~Hz}, I_{O F F}=100 \mathrm{~A}$ 


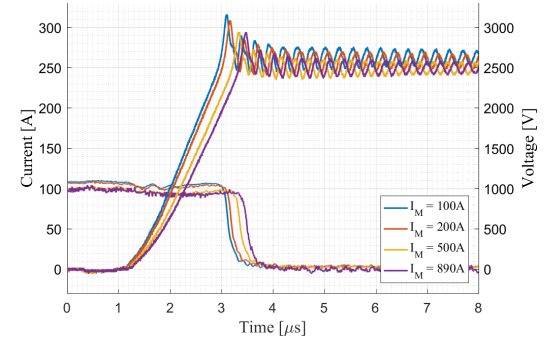

(a)

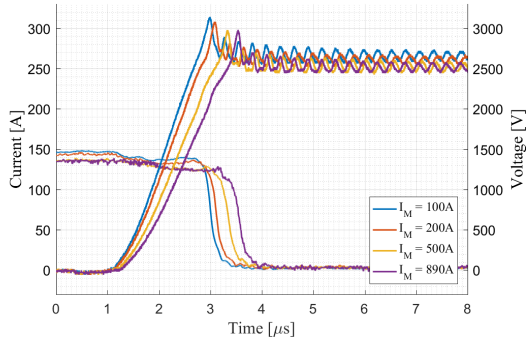

(b)

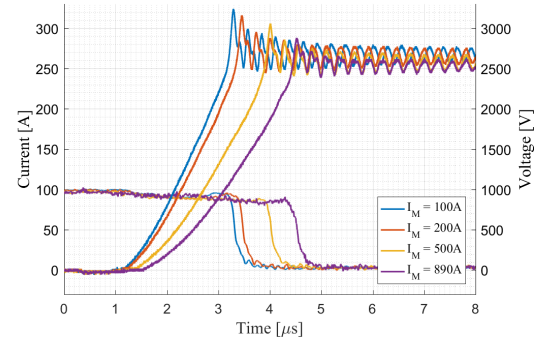

(c)

Figure 7: Turn-off current and voltage waveforms a) $f_{R}=2300 \mathrm{~Hz}, I_{O F F}=100 \mathrm{~A}$ b) $\left.f_{R}=3250, I_{O F F}=140 \mathrm{~A} \mathrm{c}\right) f_{R}=4600 \mathrm{~Hz}, I_{O F F}=100 \mathrm{~A}$

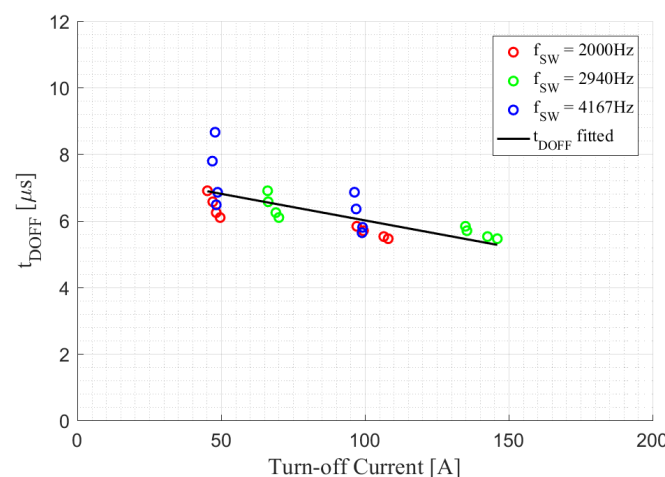

Figure 8: Measured turn-off delay times for different turn-off currents and frequencies

responding to the switching frequencies of $2000 \mathrm{~Hz}, 3000 \mathrm{~Hz}$ and $4200 \mathrm{~Hz}$ of the equivalent SRC. Resonant frequencies of the $\mathrm{L}_{\mathrm{R}}-\mathrm{C}_{\mathrm{R}}$ resonant tank are calculated to be $2300 \mathrm{~Hz}$, $3250 \mathrm{~Hz}$ and $4600 \mathrm{~Hz}$ respectively and vary slightly from these results due to the manufacturing tolerances of the passive elements.

Turn-off transient current and voltage waveforms are presented in Fig. 7 with visible ringing in the IGCT voltage due to the high parasitic inductance of the commutation loop. Referring to Fig. 8, where the turn-off delay times obtained from all of the experimental waveforms are plotted, distribution of this parameter suggests the overall trend of decreasing the $t_{D O F F}$ with increasing the turn-off current. Measurement spreads for the particular turn-off currents could be explained by the influence of the reverse recovery of the diode, $D_{2}$ in the Fig. 6 , on the turn-off process of the IGCT and further measurements are needed to investigate this behavior.

Turn-off energy losses, defined as the area under the instantaneous power curve, are shown in Fig. 9. Spread of the measured values in case of $E_{\text {off }}$ is quite narrow suggesting that only the turn-off current plays a role in defining the turnoff energy losses, regardless of the peak resonant current or the resonant frequency of the tank which is inline with the TCAD results. Values estimated form the measurement data are lower than the ones form the TCAD model due to different temperature of the device $\left(115^{\circ} \mathrm{C}\right.$ in $\mathrm{TCAD}$ and $30^{\circ} \mathrm{C}$ during the test). Having the results for the turn-off energy losses, it is easy for the designer to evaluate the turn-off power losses for the final SRC.

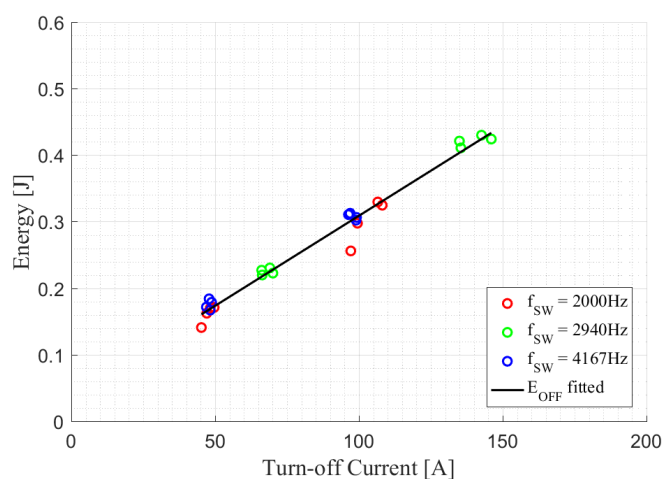

Figure 9: Calculated turn-off energy losses for different turn-off currents and frequencies

Design criteria for the dead time to be longer than $t_{D O F F}$ and shorter than the anti-parallel diode conduction time $t_{D O N}$ means that the $t_{D O F F}$ should be minimized at higher switching frequencies which implies selection of the higher turn-off currents for the SRC operation. On the other hand, higher turn-off currents cause higher turn-off switching power losses of the IGCT that further limit the switching frequency of the converter. For the practical implementation of the SRC, a trade-off between the switching frequency and the designed value of the turn-off current has to be made.

\section{Conclusion}

Estimation of the turn-off delay times and turn-off energy losses under resonant operating conditions of the IGCT are presented, supported by the TCAD simulation and measured experimental data. Relatively high resonant and switching frequency SRC operation of the high voltage semiconductor switch is assessed and limitations for the steady state operation are identified and evaluated. Requirements for the necessary dead-time are identified and the lower limit for the minimal dead time, $t_{D O F F}$ experimentally measured. Future work will include the demonstration of the continuous operation of the IGCT and investigate further limitations for the SRC operation.

\section{ACKNOWLEDGMENT}

The work presented in the paper is supported in part by the Swiss National Science Foundation under the project number 200021_165566 and in part by ABB Semiconductors, Lenzburg, Switzerland. 


\section{REFERENCES}

[1] S. Castellan, R. Menis, A. Tessarolo, and G. Sulligoi, "Power electronics for all-electric ships with MVDC power distribution system: An overview," in 2014 Ninth International Conference on Ecological Vehicles and Renewable Energies (EVER), Mar. 2014, pp. 1-7.

[2] U. Javaid, D. Dujic, and W. van der Merwe, "MVDC marine electrical distribution: Are we ready?" In IECON 2015 - 41st Annual Conference of the IEEE Industrial Electronics Society, Nov. 2015, pp. 000823 000828.

[3] H. A. B. Siddique and R. W. D. Doncker, "Evaluation of dc collector-grid configurations for large photovoltaic parks," IEEE Transactions on Power Delivery, vol. 33, no. 1, pp. 311-320, Feb. 2018.

[4] C. Dincan, P. Kjaer, Y. Chen, S. Munk-Nielsen, and C. L. Bak, "Analysis of a high-power, resonant DC-DC converter for DC wind turbines," IEEE Transactions on Power Electronics, vol. 33, no. 9, pp. 7438-7454, Sep. 2018.

[5] C. Dincan, P. Kjaer, Y. Chen, S. Nielsen, and C. L. Bak, "Selection of DC/DC converter for offshore wind farms with MVDC power collection," in 2017 19th European Conference on Power Electronics and Applications (EPE'17 ECCE Europe), Sep. 2017, P.1-P.10.

[6] S. Kenzelmann, A. Rufer, D. Dujic, F. Canales, and Y. R. De Novaes, "Isolated DC/DC structure based on modular multilevel converter," IEEE Transactions on Power Electronics, vol. 30, no. 1, pp. 89-98, 2015.

[7] D. Dujic, S. Lewdeni-Schmid, A. Mester, C. Zhao, M. Weiss, J. Steinke, M. Pellerin, and T. Chaudhuri, "Experimental characterization of LLC resonant DC/DC converter for medium voltage applications," in Proceedings of the PCIM Europe 2011, 2011, pp. 265-271.

[8] M. Stieneker and R. W. D. Doncker, "Dual-active bridge DC-DC converter systems for medium-voltage DC distribution grids," in 2015 IEEE 13th Brazilian Power Electronics Conference and 1st Southern Power Electronics Conference (COBEP/SPEC), Nov. 2015, pp. 1-6.

[9] D. Dujic, F. Kieferndorf, F. Canales, and U. Drofenik, "Power electronic traction transformer technology," in
Power Electronics and Motion Control Conference (IPEMC), 2012 7th International, IEEE, vol. 1, 2012, pp. 636-642.

[10] P. K. Steimer, H. Gruning, J. Werninger, E. Carroll, S. Klaka, and S. Linder, "IGCT - a new emerging technology for high power, low cost inverters," IEEE Industry Applications Magazine, vol. 5, no. 4, pp. 1218, 1999.

[11] Y. Suh and P. K. Steimer, "Application of IGCT in high-power rectifiers," IEEE Transactions on Industry Applications, vol. 45, no. 5, pp. 1628-1636, 2009.

[12] U. Vemulapati, M. Rahimo, M. Arnold, T. Wikström, J. Vobecky, B. Backlund, and T. Stiasny, "Recent advancements in IGCT technologies for high power electronics applications," in Power Electronics and Applications (EPE'15 ECCE-Europe), 2015 17th European Conference on, IEEE, 2015, pp. 1-10.

[13] R. Alvarez, F. Filsecker, M. Buschendorf, and S. Bernet, "Characterization of $4.5 \mathrm{kv} / 2.4 \mathrm{ka}$ press pack igbt including comparison with igct," in 2013 IEEE Energy Conversion Congress and Exposition, Sep. 2013, pp. 260-267.

[14] F. Filsecker, R. Alvarez, and S. Bernet, "Comparison of 4.5-kv press-pack igbts and igcts for medium-voltage converters," IEEE Transactions on Industrial Electronics, vol. 60, no. 2, pp. 440-449, Feb. 2013.

[15] Z. Chen, Z. Yu, X. Zhang, T. Wei, G. Lyu, L. Qu, Y. Huang, and R. Zeng, "Analysis and experiments for igbt, iegt, and igct in hybrid dc circuit breaker," IEEE Transactions on Industrial Electronics, vol. 65, no. 4, pp. 2883-2892, Apr. 2018.

[16] D. Stamenkovic, U. R. Vemulapati, M. Rahimo, T. Stiasny, and D. Dujic, "IGCT switching behaviour under low current conditions," in PCIM Europe 2018; International Exhibition and Conference for Power Electronics, Intelligent Motion, Renewable Energy and Energy Management, Jun. 2018, pp. 1-6.

[17] D. Stamenkovic, D. Dujic, M. Rahimo, U. R. Vemulapati, and T. Stiasny, "IGCT switching behaviour under resonant operating conditions," in 2018 20th European Conference on Power Electronics and Applications (EPE'18 ECCE Europe), Sep. 2018, P.1-P.9. 